\title{
ON THE DIRECT PRODUCT CONJECTURE FOR SIGMA INVARIANTS
}

\author{
DIRK SCHÜTZ
}

\begin{abstract}
We show that the direct product conjecture for $\Sigma^{n}(G ; \mathbb{Z})$, where $G$ is the direct product of two groups of type $F P_{n}$ holds for $n=3$ and give counterexamples for $n \geq 4$. Previously counterexamples were only known for a related conjecture involving the homotopical $\Sigma$-invariants, where the conjecture already fails for $n \geq 3$.
\end{abstract}

\section{INTRODUCTION}

A group $G$ is said to be of type $F P_{n}$, if there is a resolution

$$
\ldots \longrightarrow F_{i} \longrightarrow F_{i-1} \longrightarrow \ldots \longrightarrow F_{0} \longrightarrow \mathbb{Z} \longrightarrow 0
$$

of free $\mathbb{Z} G$-modules such that $F_{i}$ is finitely generated for $i \leq n$. Here $\mathbb{Z}$ is considered as a trivial $\mathbb{Z} G$-module. Bieri and Renz [5], building on work of [4], have introduced certain geometric invariants of $G$ which contain information on such finiteness properties of subgroups of $G$ which occur as kernels of real-valued homomorphisms.

Given a group of type $F P_{n}$ with $n \geq 1$, these invariants are defined as follows. We first define

$$
S(G)=(\operatorname{Hom}(G, \mathbb{R})-\{0\}) / \mathbb{R}_{+},
$$

that is, we identify nonzero homomorphisms, if one is a positive multiple of the other. This is a sphere of dimension $\operatorname{rank}(G /[G, G])-1$. If $\chi: G \rightarrow \mathbb{R}$ is a nonzero homomorphism, we still write $\chi \in S(G)$.

Given such $\chi$, we let $G_{\chi}=\{g \in G \mid \chi(g) \geq 0\}$. If there is a resolution (1) of free $\mathbb{Z} G_{\chi}$ modules with $F_{i}$ finitely generated for $i \leq k$, we say $G_{\chi}$ is of type $F P_{k}$. We now set

$$
\Sigma^{k}(G ; \mathbb{Z})=\left\{\chi \in S(G) \mid G_{\chi} \text { is of type } F P_{k}\right\} .
$$

If $G=G_{1} \times G_{2}$ and $\chi: G \rightarrow \mathbb{R}$ a homomorphism, we obtain homomorphisms $\chi_{i}: G_{i} \rightarrow \mathbb{R}$ for $i=1,2$ by restriction. For a direct product of groups the following conjecture has been formulated, see Bieri [2] or Meinert [9]. Notice that the zero homomorphism is not contained in any $\Sigma^{n}(G ; \mathbb{Z})$.

Conjecture 1. Let $G_{1}$ and $G_{2}$ be groups of type $F P_{n}$. Then $\chi \notin \Sigma^{n}\left(G_{1} \times G_{2} ; \mathbb{Z}\right)$ if and only if $\chi_{1} \notin \Sigma^{p}\left(G_{1} ; \mathbb{Z}\right)$ and $\chi_{2} \notin \Sigma^{q}\left(G_{2} ; \mathbb{Z}\right)$ for some $p$ and $q$ with $p+q=n$.

2000 Mathematics Subject Classification. Primary 20J05; Secondary 20F65, 57M07.

Key words and phrases. Sigma invariants, Novikov homology. 
For $n=1$ this is proven in Bieri, Neumann and Strebel [4] and the case $n=2$ has been established by Gehrke [7]. Gehrke [7], based on work of Meinert, also showed that $\chi \notin \Sigma^{n}\left(G_{1} \times G_{2} ; \mathbb{Z}\right)$ implies $\chi_{1} \notin \Sigma^{p}\left(G_{1} ; \mathbb{Z}\right)$ and $\chi_{2} \notin \Sigma^{q}\left(G_{2} ; \mathbb{Z}\right)$ for some $p$ and $q$ with $p+q=n$ for all $n$.

If for a given $\chi_{1}: G_{1} \rightarrow \mathbb{R}$ and $\chi_{2}: G_{2} \rightarrow \mathbb{R}$ Conjecture 1 holds, we say that $\chi_{1}$ and $\chi_{2}$ satisfy the product formula.

The conjecture is often expressed in terms of subsets of spheres as, for example, in $[2,8,9]$. It then takes the following form. Here $\Sigma^{n}(G ; \mathbb{Z})^{c}$ denotes the complement of $\Sigma^{n}(G ; \mathbb{Z})$ in $S(G)$.

Conjecture 2. Let $G_{1}$ and $G_{2}$ be groups of type $F P_{n}$. Then

$$
\Sigma^{n}\left(G_{1} \times G_{2} ; \mathbb{Z}\right)^{c}=\bigcup_{p+q=n} \Sigma^{p}\left(G_{1} ; \mathbb{Z}\right)^{c} * \Sigma^{q}\left(G_{2} ; \mathbb{Z}\right)^{c}
$$

where $*$ stands for the join in $S\left(G_{1} \times G_{2}\right)=S\left(G_{1}\right) * S\left(G_{2}\right)$.

A similar conjecture involving homotopical sigma invariants $\Sigma^{n}(G)$, see [5, Rmk.6.5] for the definition, has been shown to be false for $n \geq 3$ by Meier, Meinert and van Wyk $[8, \S 6]$. Again this conjecture was known to hold for $n \leq 2$.

The example of [8] exploits the subtle differences between groups of type $F P_{m}$ and groups of type $F_{m}$, that is, groups for which there exists a $K(G, 1)$ with finite $m$ skeleton, which do not apply in the homological version. The following Theorem now settles Conjecture 1 .

Theorem 1.1. Conjecture 1 is true for $n \leq 3$, but there exist counterexamples for $n \geq 4$.

Notice that the conjecture holds for $n=3$ despite the counterexamples to the homotopical conjecture for $n=3$. The counterexamples for $n \geq 4$ are, as the examples of [8], based on the work of Bestvina and Brady [1] on right-angled Artin groups. They are described in Example 3.7.

The proof of Theorem 1.1 is based on a description of the Sigma-invariants in terms of vanishing Novikov homology groups. This is explained in Section 2. Using a Künneth formula for these homology groups we obtain vanishing and non-vanishing results for the Novikov homology of the direct product $G_{1} \times G_{2}$. In particular, this gives an alternative approach to the results of Gehrke and Meinert mentioned above. Finally, in the last section we consider the case where one of the groups is a right-angled Artin group and describe conditions on this group so that the product formula holds.

In [2, Thm.3.2], Bieri and Geoghegan announce a result which gives an alternative proof of the case $n=3$, though the proof has yet to appear. It is worth pointing out that in [2] they also announce a proof of Conjecture 1 in case $\mathbb{Z}$ is replaced by a field k. The author would like to thank Robert Bieri and Ross Geoghegan for valuable comments and information about their unpublished work.

\section{Homological CRIteria For Sigma invariants}

In this section we want to describe a criterion for the sigma invariants based on Novikov homology. We start with the definition of the Novikov ring. 
Let $G$ be a group and $\chi: G \rightarrow \mathbb{R}$ a homomorphism. We denote by $\mathbb{Z}^{G}$ the abelian group of all functions $\lambda: G \rightarrow \mathbb{Z}$. For $\lambda \in \mathbb{Z}^{G}$ denote $\operatorname{supp} \lambda=\{g \in G \mid \lambda(g) \neq 0\}$.

Definition 2.1. The Novikov ring $\widehat{\mathbb{Z G}}_{\chi}$ is defined as

$$
\widehat{\mathbb{Z}}_{\chi}=\left\{\lambda \in \mathbb{Z}^{G} \mid \forall r \in \mathbb{R} \quad \operatorname{supp} \lambda \cap \xi^{-1}((-\infty, r]) \text { is finite }\right\}
$$

The multiplication is given by the extension of the multiplication of the group ring. If $G_{1}$ and $G_{2}$ are groups, and $G=G_{1} \times G_{2}$, it is clear that $\mathbb{Z} G=\mathbb{Z} G_{1} \otimes \mathbb{Z} G_{2}$. Now if $\chi_{i}: G_{i} \rightarrow \mathbb{R}$ are homomorphisms for $i=1,2$, we can form $\chi: G \rightarrow \mathbb{R}$ by $\chi\left(g_{1}, g_{2}\right)=\chi_{1}\left(g_{1}\right)+\chi_{2}\left(g_{2}\right)$. There is an inclusion $\widehat{\mathbb{Z} G}_{1 \chi_{1}} \otimes \widehat{\mathbb{Z} G}_{2 \chi_{2}} \rightarrow \widehat{\mathbb{Z} G}_{\chi}$, but this is in general not an isomorphism. In particular if both homomorphisms are nonzero, it is not an isomorphism. This will lead to some extra complication in Section 4 .

The following lemma is well-known, a proof can be found in Bieri [3, Thm.A.1].

Lemma 2.2. Let $G$ be a group of type $F P_{n}$ and $k \leq n$. Then the following are equivalent.

(1) $\chi \in \Sigma^{k}(G ; \mathbb{Z})$.

(2) $\operatorname{Tor}_{i}^{\mathbb{Z} G}(\widehat{\mathbb{Z} G}, \mathbb{Z})=0$ for $i \leq k$.

If $X$ is a $K(G, 1)$, we also write $H_{i}\left(X ; \widehat{\mathbb{Z} G}_{\chi}\right)=\operatorname{Tor}_{i}^{\mathbb{Z} G}\left(\widehat{\mathbb{Z G}}_{\chi}, \mathbb{Z}\right)$ for $i \in \mathbb{Z}$.

\section{The Künneth Formula For Novikov homology}

As $\widehat{\mathbb{Z} G}_{\chi} \neq \widehat{\mathbb{Z} G}_{1 \chi_{1}} \otimes \widehat{\mathbb{Z} G}_{2 \chi_{2}}$, we need a lemma on change of coefficients.

Lemma 3.1. Let $R$ be a ring and $C_{*}$ a left chain complex over $R$ of flat modules with $C_{i}=0$ for $i<0$ and $\rho: R \rightarrow S$ a ring homomorphism. If $H_{i}(C)=0$ for $i<k$, then $H_{i}\left(S \otimes_{R} C\right)=0$ for $i<k$ and $H_{k}\left(S \otimes_{R} C\right)=S \otimes_{R} H_{k}(C)$.

Proof. This follows directly from the Universal Coefficient Spectral Sequence.

Lemma 3.2. Let $C_{*}$ be a free left chain complex over $R_{1}$ with $C_{i}=0$ for $i<0$ and $D_{*}$ a free left chain complex over $R_{2}$ with $D_{i}=0$ for $i<0$. Assume also that $R_{1}$ or $R_{2}$ is torsionfree as an abelian group. If $H_{i}(C)=0$ for $i<k$ and $H_{i}(D)=0$ for $i<l$, then $H_{i}\left(C \otimes_{\mathbb{Z}} D\right)=0$ for $i<k+l$ and

$$
H_{k+l}\left(C \otimes_{\mathbb{Z}} D\right)=H_{k}(C) \otimes_{\mathbb{Z}} H_{l}(D)
$$

as $R_{1} \otimes_{\mathbb{Z}} R_{2}$-modules.

Proof. As one of $R_{1}$ or $R_{2}$ is a torsionfree abelian group, the result in terms of abelian groups follows from the ordinary Künneth theorem. Furthermore, the resulting isomorphism of abelian groups

$$
H_{k}(C) \otimes_{\mathbb{Z}} H_{l}(D) \longrightarrow H_{k+l}\left(C \otimes_{\mathbb{Z}} D\right)
$$

is easily seen to respect the $R_{1} \otimes_{\mathbb{Z}} R_{2}$-module structure. This gives the result.

Corollary 3.3. Let $G_{1}$ be a group of type $F P_{k}$ and $G_{2}$ be a group of type $F P_{l}$ with $k, l$ positive integers. Let $\chi: G_{1} \times G_{2} \rightarrow \mathbb{R}$ be a homomorphism and $\chi_{i}$ the 
restriction to $G_{i}$ for $i=1,2$. Assume that $\chi_{1} \in \Sigma^{k-1}\left(G_{1} ; \mathbb{Z}\right)$ and $\chi_{2} \in \Sigma^{l-1}\left(G_{2} ; \mathbb{Z}\right)$. Then

$$
\begin{aligned}
\operatorname{Tor}_{i}^{\mathbb{Z} G}(\widehat{\mathbb{Z} G}, \mathbb{Z}) & =0 \quad \text { for } i \leq k+l-1 \\
\operatorname{Tor}_{k+l}^{\mathbb{Z} G}\left(\widehat{\mathbb{Z} G}_{\chi}, \mathbb{Z}\right) & \cong \widehat{\mathbb{Z} G}_{\chi} \otimes_{R}\left(\operatorname{Tor}_{k+l}^{\mathbb{Z} G_{1}}\left(\widehat{\mathbb{Z} G_{1}}, \mathbb{Z}_{\chi_{1}}\right) \otimes_{\mathbb{Z}} \operatorname{Tor}_{k+l}^{\mathbb{Z} G_{2}}\left(\widehat{\mathbb{Z} G_{2}}, \mathbb{Z}_{\chi_{2}}\right)\right)
\end{aligned}
$$

where $G=G_{1} \times G_{2}$ and $R=\widehat{\mathbb{Z} G}_{1 \chi_{1}} \otimes \widehat{\mathbb{Z} G}_{2 \chi_{2}}$.

Proof. If $C \rightarrow \mathbb{Z}$ is a resolution of $\mathbb{Z}$ of free $\mathbb{Z} G_{1}$-modules and $D \rightarrow \mathbb{Z}$ a resolution of free $\mathbb{Z} G_{2}$-modules, the double complex $C \otimes_{\mathbb{Z}} D$ gives a free resolution of $\mathbb{Z}$ over $\mathbb{Z} G$. The result then follows from Lemma 2.2, Lemma 3.2 and Lemma 3.1 as all of the involved rings are torsionfree as abelian groups.

Let us also state a version of Corollary 3.3 in the case that $G_{1}$ and $G_{2}$ are of type $F_{n}$.

Corollary 3.4. Let $X$ be a $K\left(G_{1}, 1\right)$ and $Y$ a $K\left(G_{2}, 1\right)$, both with finite $n$-skeleton, and let $k, l$ be positive integers with $k+l \leq n$. Let $\chi: G_{1} \times G_{2} \rightarrow \mathbb{R}$ be a homomorphism and $\chi_{i}$ the restriction to $G_{i}$ for $i=1,2$. Assume that $\chi_{1} \in \Sigma^{k-1}\left(G_{1} ; \mathbb{Z}\right)$ and $\chi_{2} \in \Sigma^{l-1}\left(G_{2} ; \mathbb{Z}\right)$. Then $\chi \in \Sigma^{k+l-1}(G ; \mathbb{Z})$ and

$$
H_{k+l}\left(X \times Y ; \widehat{\mathbb{Z}}_{\chi}\right) \cong \widehat{\mathbb{Z}}_{\chi} \otimes_{R}\left(H_{k}\left(X ; \widehat{\mathbb{Z} G}_{\chi_{\chi_{1}}}\right) \otimes_{\mathbb{Z}} H_{l}\left(Y ; \widehat{\mathbb{Z} G}_{\chi_{\chi_{2}}}\right)\right)
$$

where $G=G_{1} \times G_{2}$ and $R=\widehat{\mathbb{Z} G}_{\chi_{\chi_{1}}} \otimes \widehat{\mathbb{Z} G}_{2 \chi_{2}}$.

Proof. The Eilenberg-Zilber chain map $C_{*}(\tilde{X}) \otimes_{\mathbb{Z}} C_{*}(\tilde{Y}) \rightarrow C_{*}(\tilde{X} \times \tilde{Y})$, where $\tilde{X}$ and $\tilde{Y}$ are the universal covering spaces of $X$ and $Y$, is a chain homotopy equivalence of $\mathbb{Z} G$ chain complexes, so

$$
H_{*}\left(X \times Y ;{\widehat{\mathbb{Z} G_{1}}}_{\chi_{1}} \otimes{\widehat{\mathbb{Z} G_{2}}}_{\chi_{2}}\right) \cong H_{*}\left(C\left(X ; \widehat{\mathbb{Z} G_{1}} \chi_{1}\right) \otimes C\left(Y ;{\widehat{\mathbb{Z} G_{2}}}_{\chi_{2}}\right)\right) .
$$

Therefore the result follows from Lemma 3.2 and Lemma 3.1 as in Corollary 3.3.

Corollary 3.3 gives an alternative proof of one implication of Conjecture 1, see Gehrke [7] for a different proof. It also indicates why the conjecture is not true in general: a tensor product of two non-trivial abelian groups can be trivial.

Corollary 3.5. Let $G_{1}$ and $G_{2}$ be groups of type $F P_{n}$. Assume $\chi \notin \Sigma^{n}\left(G_{1} \times G_{2} ; \mathbb{Z}\right)$. Then $\chi_{1} \notin \Sigma^{p}\left(G_{1} ; \mathbb{Z}\right)$ and $\chi_{2} \notin \Sigma^{q}\left(G_{2} ; \mathbb{Z}\right)$ for some $p$ and $q$ with $p+q=n$.

Proof. Choose $p$ with $\chi_{1} \in \Sigma^{p-1}\left(G_{1} ; \mathbb{Z}\right)-\Sigma^{p}\left(G_{1} ; \mathbb{Z}\right)$ and $q$ with $\chi_{2} \in \Sigma^{q-1}\left(G_{2} ; \mathbb{Z}\right)-$ $\Sigma^{q}\left(G_{2} ; \mathbb{Z}\right)$. Then $\chi \in \Sigma^{p+q-1}\left(G_{1} \times G_{2} ; \mathbb{Z}\right)$ by Corollary 3.3 , so $p+q \leq n$. If $p+q<n$ replace $p$ by $n-q>p$.

Let $X$ be a finite CW-complex and $G=\pi_{1}(X)$. Given a nonzero homomorphism $\chi: G \rightarrow \mathbb{R}$ we can find a map $f: \tilde{X} \rightarrow \mathbb{R}$, where $\tilde{X}$ is the universal cover of $X$, satisfying $f(g x)=\chi(g)+f(x)$ for all $x \in \tilde{X}$ and $g \in G$. In this situation, we denote $N=f^{-1}([0, \infty))$.

The following Lemma is due to Sikorav [10], it follows from the fact that the Novikov ring can be interpreted as an inverse limit, compare also the proof of Lemma 4.2 below. 
Lemma 3.6. Let $X$ be a finite $C W$ complex, $\chi: \pi_{1}(X) \rightarrow \mathbb{R}$ a nonzero homomorphism, $\rho: \tilde{X} \rightarrow X$ the universal covering space and $G=\pi_{1}(X)$. Let $g \in G$ satisfy $\xi(g)>0$ and let $q \in \mathbb{Z}$. Then there is a natural short exact sequence

(2) $\quad 0 \longrightarrow \lim _{\leftarrow}^{1} H_{q+1}\left(\tilde{X}, g^{i} N ; \mathbb{Z}\right) \longrightarrow H_{q}\left(X ; \widehat{\mathbb{Z} G}_{\chi}\right) \longrightarrow \lim _{\leftarrow} H_{q}\left(\tilde{X}, g^{i} N ; \mathbb{Z}\right) \longrightarrow 0$

where the limits are taken over positive integers $i$ and the maps are induced by inclusions.

Given a simplicial complex $L$ which is a flag complex, denote $L^{0}$ as the set of vertices and $L^{1}$ as the set of edges. Bestvina and Brady [1] define the right-angled Artin group corresponding to $L$ by

$$
\left.G_{L}=\left\langle v_{i} \in L^{0}\right|\left[v_{i}, v_{j}\right] \text { for }\left(v_{i}, v_{j}\right) \in L^{1}\right\rangle .
$$

Example 3.7. Let $p$ and $q$ be positive integers with $(p, q)=1$ and $n, m \geq 2$. Let $L_{1}=M(\mathbb{Z} / p, n-1)$ and $L_{2}=M(\mathbb{Z} / q, m-1)$ be Moore spaces, that is, $L_{1}$ is obtained from the $n-1$-sphere by attaching an $n$-cell with a map of degree $p$ and similar for $L_{2}$. Subdivide $L_{1}$ and $L_{2}$ so that they are flag complexes. For $i=1,2$ let $G_{i}=G_{L_{i}}$ be the associated right angled Artin group and $\chi_{i}: G_{i} \rightarrow \mathbb{Z}$ the homomorphism sending every generator to 1 . There exists a finite $K\left(G_{i}, 1\right)$ which we denote by $Q_{i}$ for $i=1,2$, see, for example, [1]. By Bestvina and Brady [1, Thm.7.1] together with Lemma 3.6 we get

$$
H_{i}\left(Q_{1} ; \widehat{\mathbb{Z} G}_{1 \chi_{1}}\right) \cong\left\{\begin{array}{cl}
0 & \text { for } \quad i \neq n \\
\lim \bigoplus \mathbb{Z} / p & \text { for } i=n
\end{array}\right.
$$

and

$$
H_{i}\left(Q_{2} ; \widehat{\mathbb{Z} G}_{2 \chi_{2}}\right) \cong\left\{\begin{array}{cl}
0 & \text { for } \quad i \neq m \\
\lim _{\leftarrow} \bigoplus \mathbb{Z} / q & \text { for } i=m
\end{array}\right.
$$

where both inverse systems are given by projections onto direct summands. In particular, we get that $H_{n}\left(Q_{1} ; \widehat{\mathbb{Z} G}_{1 \chi_{1}}\right) \neq 0$ and is $p$-torsion as an abelian group, while $H_{m}\left(Q_{2} ; \widehat{\mathbb{Z}}_{2 \chi_{2}}\right) \neq 0$ and is $q$-torsion as an abelian group. As $(p, q)=1$, we get $H_{n+m}\left(Q_{1} \times Q_{2} ; \widetilde{\mathbb{Z} G_{1} \times G_{2 \chi_{1}+\chi_{2}}}\right)=0$ from Corollary 3.4. Therefore we have a counterexample to Conjecture 1 for $n \geq 4$.

Remark 3.8. Notice that $G=G_{1} \times G_{2}$ in Example 3.7 is the right-angled Artin group corresponding to the join $L=L_{1} * L_{2}$. It is easy to see that $L$ is contractible, so the counterexample can be derived directly from the work of Bestvina and Brady. Nevertheless the Künneth formula will become useful in the next section.

\section{Proof of the Conjecture for $n=3$}

We were able to find a counterexample by producing non-zero Novikov homology groups whose tensor product was zero. In this section we will see that this is not possible if a first Novikov homology group is non-zero.

Let $G$ be a group and $C \rightarrow \mathbb{Z}$ be a free resolution over $\mathbb{Z} G$ with $C_{i}$ finitely generated for $i \leq k$. By Bieri and Renz [5, Lm.3.1] there is a subcomplex $C^{+} \subset C$ of $\mathbb{Z} G_{\chi^{-}}$ modules which is finitely generated free for $i \leq k$ and such that the rank of $C_{i}^{+}$ equals the rank of $C_{i}$. Given a $g \in G$ with $\chi(g)>0$ we can now form an inverse system $H_{*}\left(C / g^{i} C^{+}\right) \longleftarrow H_{*}\left(C / g^{i+j} C^{+}\right)$with $i, j \geq 0$. 
Lemma 4.1. Let $G$ and $C$ be as above. If $\chi \in \Sigma^{k-1}(G ; \mathbb{Z})-\Sigma^{k}(G ; \mathbb{Z})$, then the inverse limit $\lim H_{k}\left(C / g^{i} C^{+}\right)$is non-trivial, where $g \in G$ satisfies $\chi(g)>0$.

Proof. By [5, Thm.3.2] we get that for every $i$ there is an $x_{i} \in \tilde{H}_{k-1}\left(g^{i} C^{+}\right)$with $0 \neq j_{*} x_{i} \in \tilde{H}_{k-1}\left(C^{+}\right)$. But by the Sigma criterion [5, Thm.C(III)], $x_{i}$ can be represented in any $\tilde{H}_{k-1}\left(g^{m} C^{+}\right)$with $m \geq i$. Therefore there is a non-trivial element in $\lim _{\leftarrow} \tilde{H}_{k-1}\left(g^{i} C^{+}\right) \cong \lim _{\leftarrow} H_{k}\left(C / g^{i} C^{+}\right)$.

Lemma 4.2. In the situation of Lemma 4.1 there is a surjective homomorphism

$$
\operatorname{Tor}_{k}^{\mathbb{Z} G}\left(\widehat{\mathbb{Z} G}_{\chi}, \mathbb{Z}\right) \longrightarrow \lim _{\leftarrow} H_{k}\left(C / g^{i} C^{+}\right)
$$

of abelian groups.

Proof. For $g \in G$ with $\chi(g)>0$ there is an inverse system of abelian groups $\mathbb{Z} G / g^{i} \mathbb{Z} G_{\chi} \longleftarrow \mathbb{Z} G / g^{j+i} \mathbb{Z} G_{\chi}$ whose inverse limit is exactly $\widehat{\mathbb{Z}}_{\chi}$. If $C_{l}$ is a finitely generated free $\mathbb{Z} G$-module, then $\lim _{\leftarrow} C_{l} / g^{i} C_{l}^{+}=\widehat{\mathbb{Z}}_{\chi} \otimes_{\mathbb{Z} G} C_{l}$. We get the standard short exact sequence

$$
0 \longrightarrow \lim _{\leftarrow}^{1} H_{l+1}\left(C / g^{i} C^{+}\right) \longrightarrow H_{l}\left(\lim _{\leftarrow} C / g^{i} C^{+}\right) \longrightarrow \lim _{\leftarrow} H_{l}\left(C / g^{i} C^{+}\right) \longrightarrow 0
$$

and as we have that $C_{l}$ is finitely generated for $l \leq k$ we get a surjective homomorphism $\operatorname{Tor}_{k}^{\mathbb{Z} G}\left(\widehat{\mathbb{Z} G}_{\chi}, \mathbb{Z}\right) \rightarrow \lim _{\leftarrow} H_{k}\left(C / g^{i} C^{+}\right)$. Notice that there is only an inclusion $\widehat{\mathbb{Z} G}_{\chi} \otimes_{\mathbb{Z} G} C_{k+1} \rightarrow \lim _{\leftarrow} C_{k+1} / g^{i} C_{k+1}^{+}$in the case that $C_{k+1}$ is not finitely generated, but we still get a surjection $\operatorname{Tor}_{k}^{\mathbb{Z} G}\left(\widehat{\mathbb{Z} G}_{\chi}, \mathbb{Z}\right) \rightarrow H_{k}\left(\underset{\leftarrow}{\lim C / g^{i} C^{+}}\right)$. The Lemma follows.

As $\lim H_{k}\left(C / g^{i} C^{+}\right) \neq 0$ by Lemma 4 .1, we get a non-trivial homomorphism $\operatorname{Tor}_{k}^{\overleftarrow{Z} G}(\widehat{\mathbb{Z} G}, \mathbb{Z}) \rightarrow H_{k}\left(C / C^{+}\right)$of abelian groups.

Proposition 4.3. For $i=1,2$ let $G_{i}$ be a group of type $F P_{k_{i}}$ for some $k_{i} \geq 1$, and $\chi_{i}: G_{i} \rightarrow \mathbb{R}$ a nonzero homomorphism with $\chi_{i} \in \Sigma^{k_{i}-1}\left(G_{i} ; \mathbb{Z}\right)-\Sigma^{k_{i}}\left(G_{i} ; \mathbb{Z}\right)$. Assume that there is a non-trivial abelian group homomorphism $\varphi_{1}: \operatorname{Tor}_{k_{1}}^{\mathbb{Z} G_{1}}\left(\widehat{\mathbb{Z} G_{1}}, \mathbb{Z}\right) \rightarrow \mathbb{Z}$ which factors through $\mathrm{H}_{k_{1}}\left(\mathrm{C} / \mathrm{C}^{+}\right)$. Then

$$
\begin{aligned}
\operatorname{Tor}_{i}^{\mathbb{Z} G}\left(\widehat{\mathbb{Z} G}_{\chi}, \mathbb{Z}\right) & =0 \quad \text { for } i \leq k_{1}+k_{2}-1 \\
\operatorname{Tor}_{k_{1}+k_{2}}^{\mathbb{Z} G}\left(\widehat{\mathbb{Z} G}_{\chi}, \mathbb{Z}\right) & \neq 0
\end{aligned}
$$

where $G=G_{1} \times G_{2}$ and $\chi: G \rightarrow \mathbb{R}$ is the sum of $\chi_{1}$ and $\chi_{2}$.

Proof. It follows from Corollary 3.3 that $\operatorname{Tor}_{i}^{\mathbb{Z} G}(\widehat{\mathbb{Z} G}, \mathbb{Z})=0$ for $i \leq k$, so we need to show that $\operatorname{Tor}_{k+1}^{\mathbb{Z} G}(\widehat{\mathbb{Z} G}, \mathbb{Z}) \neq 0$.

By assumption $\varphi_{1}: \operatorname{Tor}_{k_{1}}^{\mathbb{Z} G_{1}}\left(\widehat{\mathbb{Z} G_{1}} \chi_{1}, \mathbb{Z}\right) \rightarrow \mathbb{Z}$ factors through $H_{k_{1}}\left(C / C^{+}\right)$. Denote the image in $H_{k_{1}}\left(C / C^{+}\right)$by $A_{1}$. Furthermore, there is a non-trivial homomorphism $\varphi_{2}: \operatorname{Tor}_{k}^{\mathbb{Z} G_{2}}\left(\widehat{\mathbb{Z} G}_{2 \chi_{2}}, \mathbb{Z}\right) \rightarrow H_{k}\left(D / D^{+}\right)$, where $D \rightarrow \mathbb{Z}$ is a free resolution over $\mathbb{Z} G_{2}$. Let us denote the image by $A_{2}$. These homomorphisms combine to an epimorphism

$$
\varphi: \operatorname{Tor}_{k_{1}}^{\mathbb{Z} G_{1}}\left(\widehat{\mathbb{Z} G}_{\chi_{1}}, \mathbb{Z}\right) \otimes_{\mathbb{Z}} \operatorname{Tor}_{k_{2}}^{\mathbb{Z} G_{2}}\left(\widehat{\mathbb{Z} G}_{2 \chi_{2}}, \mathbb{Z}\right) \rightarrow A_{1} \otimes A_{2}
$$

of abelian groups. 
Given $x \in \operatorname{Tor}_{k_{1}}^{\mathbb{Z} G_{1}}\left(\widehat{\mathbb{Z} G}_{\chi_{1}}, \mathbb{Z}\right)$ and $y \in \operatorname{Tor}_{k_{2}}^{\mathbb{Z} G_{2}}\left({\widehat{\mathbb{Z} G_{2}}}_{\chi_{2}}, \mathbb{Z}\right)$, there exists an $M>0$ such that

$$
\varphi\left(g_{1} x \otimes g_{2} y\right)=0, \text { if } \chi_{1}\left(g_{1}\right)+\chi_{2}\left(g_{2}\right) \geq M
$$

To see this notice that there is $M>0$ such that $\varphi_{1}\left(g_{1} x\right)=0 \in H_{k_{1}}\left(C / C^{+}\right)$if $\chi_{1}(g) \geq M / 2$ as the support of a cycle representing $x$ will be contained in $N$ if $\chi_{1}\left(g_{1}\right)$ is big enough. Here notice that $C / C^{+} \cong\left(\widehat{\mathbb{Z} G}_{\chi_{1}} \otimes_{\mathbb{Z} G_{1}} C\right) /\left({\widehat{\mathbb{Z} G_{1 \chi_{1}}}}_{\chi_{\mathbb{Z} G_{1 \chi_{1}}}}^{+} C^{+}\right)$, where

$$
{\widehat{\mathbb{Z} G_{1}}}_{\chi_{1}}^{+}=\left\{\lambda \in \widehat{\mathbb{Z} G}_{\chi_{1}} \mid \operatorname{supp} \lambda \subset \chi_{1}^{-1}([0, \infty))\right\} .
$$

A similar argument gives $\varphi_{2}\left(g_{2} y\right)=0 \in H_{k}\left(D / D^{+}\right)$for $\chi_{2}\left(g_{2}\right) \geq M / 2$ big enough. Let us denote

$$
\begin{aligned}
\Lambda & =\widehat{\mathbb{Z}}_{\chi} \\
R & =\widehat{\mathbb{Z} G}_{1 \chi_{1}} \otimes_{\mathbb{Z}} \widehat{\mathbb{Z} G}_{2 \chi_{2}}
\end{aligned}
$$

If $\lambda \in \Lambda$, then there is a $\bar{\lambda} \in \mathbb{Z} G$ with $\operatorname{supp} \lambda-\bar{\lambda} \subset \chi^{-1}([M, \infty))$ for any $M>0$. Now define a homomorphism of abelian groups

$$
\psi: \Lambda \otimes_{R}\left(\operatorname{Tor}_{k_{1}}^{\mathbb{Z} G_{1}}\left(\widehat{\mathbb{Z} G}_{\chi_{1}}, \mathbb{Z}\right) \otimes_{\mathbb{Z}} \operatorname{Tor}_{k_{2}}^{\mathbb{Z} G_{2}}\left(\widehat{\mathbb{Z} G}_{2 \chi_{2}}, \mathbb{Z}\right)\right) \rightarrow A_{1} \otimes A_{2}
$$

by $\psi\left(\lambda \otimes_{R}(x \otimes y)\right)=\varphi(\bar{\lambda} \cdot(x \otimes y))$, where supp $\lambda-\bar{\lambda} \subset \chi^{-1}([M, \infty))$ with $M>0$ as in (3). This does not depend on $\bar{\lambda}$, because if $\tilde{\lambda} \in \mathbb{Z} G$ also satisfies $\operatorname{supp} \lambda-\tilde{\lambda} \subset$ $\chi^{-1}([M, \infty))$, we get $\operatorname{supp} \bar{\lambda}-\tilde{\lambda} \subset \chi^{-1}([M, \infty))$ and

$$
\varphi(\bar{\lambda} \cdot(x \otimes y))=\varphi(\tilde{\lambda} \cdot(x \otimes y))
$$

by (3). Let us show that $\psi$ is well defined, that is, for $r \in R$ let us show that

$$
\psi\left(\lambda \otimes_{R} r \cdot(x \otimes y)\right)=\psi\left(\lambda \cdot r \otimes_{R}(x \otimes y)\right) .
$$

There is a $K \leq 0$ with supp $r \cup \operatorname{supp} \lambda \subset \chi^{-1}([K, \infty))$. Let $M>0$ satisfy

$$
\varphi\left(h_{1} x \otimes h_{2} y\right)=0 \text { for } \chi\left(h_{1}, h_{2}\right) \geq M .
$$

Then

$$
\varphi\left(\left(g_{1}, g_{2}\right) \cdot r \cdot(x \otimes y)\right)=0 \text { for } \chi\left(g_{1}, g_{2}\right) \geq M-K .
$$

Write $\lambda=\bar{\lambda}+\mu$ with $\bar{\lambda} \in \mathbb{Z} G$ and $\operatorname{supp} \mu \subset \chi^{-1}([M-K, \infty))$. Also let $r=\bar{r}+\nu$ with $\bar{r} \in \mathbb{Z} G$ and $\operatorname{supp} \nu \subset \chi^{-1}([M-K, \infty))$. Notice that $\nu \in R$. Then $\lambda \cdot r=$ $\bar{\lambda} \bar{r}+\bar{\lambda} \nu+\mu \bar{r}+\mu \nu$ and supp $\lambda r-\bar{\lambda} \bar{r} \subset \chi^{-1}([M, \infty))$.

According to the definition of $\psi$ we get

$$
\begin{aligned}
\psi(\lambda \otimes r(x \otimes y)) & =\varphi(\bar{\lambda} \cdot r(x \otimes y)) \\
& =\varphi(\bar{\lambda} \bar{r}(x \otimes y)+\bar{\lambda} \nu(x \otimes y)) \\
& =\varphi(\bar{\lambda} \bar{r}(x \otimes y)) \\
& =\psi(\lambda r \otimes(x \otimes y))
\end{aligned}
$$

which shows that $\psi$ is indeed a well defined homomorphism of groups. Since $\varphi$ is surjective, the same holds for $\psi$. As there is a non-trivial homomorphism $A_{1} \rightarrow \mathbb{Z}$, it follows that $A_{1} \otimes A_{2}$ is non-trivial. By Corollary 3.3 this shows that $\operatorname{Tor}_{k_{1}+k_{2}}^{\mathbb{Z} G}\left(\widehat{\mathbb{Z} G}_{\chi}, \mathbb{Z}\right) \neq 0$. 
The assumptions of Proposition 4.3 are not always satisfied, as Example 3.7 shows, but for $k_{1}=1$ they are satisfied:

Theorem 4.4. Let $G_{1}$ and $G_{2}$ be groups of type $F P_{n}$. Assume $\chi_{1}: G_{1} \rightarrow \mathbb{R}$ is a nonzero homomorphism with $\chi_{1} \notin \Sigma^{1}\left(G_{1} ; \mathbb{Z}\right)$. If $\chi_{2} \notin \Sigma^{n-1}\left(G_{2} ; \mathbb{Z}\right)$, then $\chi=\chi_{1}+\chi_{2} \notin \Sigma^{n}\left(G_{1} \times G_{2} ; \mathbb{Z}\right)$.

Proof. We have to show that there is always a non-trivial homomorphism

$$
\operatorname{Tor}_{1}^{\mathbb{Z} G_{1}}\left(\widehat{\mathbb{Z} G}_{1 \chi_{1}}, \mathbb{Z}\right) \rightarrow \mathbb{Z}
$$

which factors through $H_{1}\left(C / C^{+}\right)$. But in degree 1 we can look at $H_{1}\left(X ; \widehat{\mathbb{Z}}_{1 \chi_{1}}\right) \rightarrow$ $H_{1}(\tilde{X}, N) \rightarrow \mathbb{Z}$, where $X$ is a connected CW-complex with $\pi_{1}(X)=G_{1}$ and finite 1-skeleton, and where $N=f^{-1}([0, \infty))$ for a map $f: \tilde{X} \rightarrow \mathbb{R}$ with $f(g x)=$ $\chi_{1}(g)+f(x)$ for all $x \in \tilde{X}$ and $g \in G_{1}$.

We can assume that $X$ has only one 0 -cell $x$ and a 1-cell for every generator of $G_{1}$ from a finite generating set.

Let $\sigma \subset \tilde{X}$ be a path in the 1-skeleton of $\tilde{X}$ which represents a nonzero element $[\sigma] \in H_{1}(\tilde{X}, N)$. We can think of $\sigma$ as a 1-chain in $\tilde{X}$ with $\partial \sigma=v-h v$, where $v \in N$ is a lift of $x$ and $h \in G_{1}$.

Let $g \in G_{1}$ be a generator that satisfies $\chi_{1}(g)>0$ and let $e \subset \tilde{X}$ be a 1-cell connecting $v$ and $g v$. We can assume that $e \subset N$ and think of $e$ as a 1-chain with $\partial e=v-g v$. Then

$$
z=\sigma-(1-g)^{-1} e+h(1-g)^{-1} e
$$

represents a cycle in $\widehat{\mathbb{Z} G}_{1 \chi_{1}} \otimes_{\mathbb{Z} G_{1}} C_{1}(\tilde{X})$ whose Novikov homology class is send to $[\sigma]$ under $\varphi_{1}: H_{1}\left(X ; \widehat{\mathbb{Z} G}_{1 \chi_{1}}\right) \rightarrow H_{1}(\tilde{X}, N)$. Now $H_{1}(\tilde{X}, N) \cong \tilde{H}_{0}(N)$ is a free abelian group and $\tilde{H}_{0}(C(v) \cup C(h v)) \cong \mathbb{Z}$ is a direct summand, where $C(v)$ and $C(h v)$ are the connected components of $v$ and $h v$ in $N$, respectively.

This gives the necessary surjection $H_{1}\left(X ; \widehat{\mathbb{Z} G}_{\chi_{1}}\right) \rightarrow \mathbb{Z}$ so the result follows from Proposition 4.3 in connection with Lemma 2.2 .

Theorem 4.5. Let $G_{1}$ and $G_{2}$ be groups of type $F P_{n}$ with $n \leq 3$. Then $\chi \notin$ $\Sigma^{n}\left(G_{1} \times G_{2} ; \mathbb{Z}\right)$ if and only if $\chi_{1} \notin \Sigma^{p}\left(G_{1} ; \mathbb{Z}\right)$ and $\chi_{2} \notin \Sigma^{q}\left(G_{2} ; \mathbb{Z}\right)$ for some $p$ and $q$ with $p+q=n$.

Proof. Let $\chi_{1} \notin \Sigma^{p}\left(G_{1} ; \mathbb{Z}\right)$ and $\chi_{2} \notin \Sigma^{q}\left(G_{2} ; \mathbb{Z}\right)$ for some $p$ and $q$ with $p+q=n$. As $n \leq 3$, we get $p \leq 1$ or $q \leq 1$. Without loss of generality we can assume $p \leq 1$. If $p=1$ we get $\chi \notin \Sigma^{r+1}(G ; \mathbb{Z})$ by Theorem 4.4 , where $r \leq q$ is such that $\chi_{2} \in \Sigma^{r-1}\left(G_{2} ; \mathbb{Z}\right)-\Sigma^{r}\left(G_{2} ; \mathbb{Z}\right)$. As $r+1 \leq q+p \leq n$, the result follows. If $p=0$, note that $\chi_{1}=0$ and

$$
\operatorname{Tor}_{0}^{\mathbb{Z} G_{1}}\left(\widehat{\mathbb{Z} G}_{1 \chi_{1}}, \mathbb{Z}\right)=\mathbb{Z}
$$

and so we get

$$
\operatorname{Tor}_{r}^{\mathbb{Z} G}\left(\widehat{\mathbb{Z} G}_{\chi}, \mathbb{Z}\right) \cong \widehat{\mathbb{Z}}_{\chi} \otimes_{R} \operatorname{Tor}_{r}^{\mathbb{Z} G_{2}}\left(\widehat{\mathbb{Z} G}_{\chi_{2}}, \mathbb{Z}\right)
$$

where $r \leq q$ satisfies $\chi_{2} \in \Sigma^{r-1}\left(G_{2} ; \mathbb{Z}\right)-\Sigma^{r}\left(G_{2} ; \mathbb{Z}\right)$ and $R=\mathbb{Z} G_{1} \otimes_{\mathbb{Z}} \widehat{\mathbb{Z}}_{2 \chi_{2}}$. By a similar argument as in the proof of Proposition 4.3 we see that this group is non-trivial. 


\section{Applications to Right-Angled Artin groups}

In this section we want to look for right-angled Artin groups which do have a nontrivial homomorphism of abelian groups $\varphi_{1}: \operatorname{Tor}_{k}^{\mathbb{Z} G}\left(\widehat{\mathbb{Z} G}_{\chi}, \mathbb{Z}\right) \rightarrow \mathbb{Z}$ which factors through $H_{k}\left(C / C^{+}\right)$, so that Proposition 4.3 does apply. In the case that $G=G_{L}$ is a right-angled Artin group we have to look for a non-trivial homomorphism

$$
H_{k}\left(X_{L} ; \widehat{\mathbb{Z} G_{L}}\right) \rightarrow H_{k}\left(\tilde{X}_{L}, N\right) \rightarrow \mathbb{Z}
$$

where $X_{L}$ is the standard $K\left(G_{L}, 1\right)$ as in [1].

Let $\tilde{X}=\tilde{X}_{L}$ be the universal cover of $X_{L}$ and for a given homomorphism $\chi: G_{L} \rightarrow$ $\mathbb{R}$ define $h: \tilde{X} \rightarrow \mathbb{R}$ as in Bux and Gonzalez [6], that is, $h$ is linear on cells. If $J \subset \mathbb{R}$ is an interval, denote $\tilde{X}_{J}=h^{-1}(J)$ and $\tilde{X}_{J}^{0}$ the union of 0-cells in $\tilde{X}_{J}$.

Proposition 5.1. Let $L$ be a finite flag complex and $G_{L}$ the corresponding rightangled Artin group. Let $\chi: G_{L} \rightarrow \mathbb{R}$ be a homomorphism with $\chi\left(v_{i}\right) \neq 0$ for all generators $v_{i}$ of $G_{L}$. Then there exists a surjection

$$
H_{k}\left(X_{L} ; \widehat{\mathbb{Z} G_{L}}\right) \longrightarrow \tilde{H}_{k-1}(L)
$$

which factors through $H_{k}\left(\tilde{X}, \tilde{X}_{[0, \infty)}\right)$ for all integers $k$.

Proof. In the case when $\chi$ is equal to 1 on every generator, this follows directly from [1, Thm.7.1]. In general we have to combine the methods of [6, Sec.1] and [1, Sec.6,7] to get that

$$
H_{*}\left(\tilde{X}, \tilde{X}_{[t, \infty)}\right) \cong \bigoplus_{v \in \tilde{X}_{(-\infty, 0)}^{0}} \tilde{H}_{*-1}(L)
$$

and that for $t<t^{\prime}$ the map $H_{*}\left(\tilde{X}, \tilde{X}_{[t, \infty)}\right) \leftarrow H_{*}\left(\tilde{X}, \tilde{X}_{\left[t^{\prime}, \infty\right)}\right)$ is the obvious projection. We omit the details. Combining with Lemma 3.6 we get the result.

Theorem 5.2. Let $k$ be an integer, and $L$ a finite flag complex with $\tilde{H}_{i}(L)=0$ for $i<k$ and $\tilde{H}_{k}(L)$ contains $\mathbb{Z}$ as a direct summand. Let $\chi_{1}: G_{L} \rightarrow \mathbb{R}$ be a homomorphism with $\chi_{1}\left(v_{i}\right) \neq 0$ for all generators $v_{i}$ of $G_{L}$. Let $G$ be a group of type $F P_{n+1}$ and $\chi_{2}: G \rightarrow \mathbb{R}$ satisfies $\chi_{2} \in \Sigma^{n-1-k}(G ; \mathbb{Z})-\Sigma^{n-k}(G ; \mathbb{Z})$. Then $\chi=\chi_{1}+\chi_{2}$ satisfies $\chi \in \Sigma^{n}\left(G_{L} \times G ; \mathbb{Z}\right)-\Sigma^{n+1}\left(G_{L} \times G ; \mathbb{Z}\right)$.

Proof. We have $\chi_{1} \in \Sigma^{k}\left(G_{L} ; \mathbb{Z}\right)-\Sigma^{k+1}\left(G_{L} ; \mathbb{Z}\right)$ and by Proposition 5.1 we can use Propositon 4.3 which implies that $\chi \in \Sigma^{n}\left(G_{L} \times G ; \mathbb{Z}\right)-\Sigma^{n+1}\left(G_{L} \times G ; \mathbb{Z}\right)$.

So with the conditions described in Theorem 5.2 on $G_{L}$ and $\chi_{1}$ we get that the product formula holds for $\chi_{1}$ and every $G$ and $\chi_{2}: G \rightarrow \mathbb{R}$. Note that $\tilde{H}_{k}(L)$ is a finitely generated abelian group, so if this group does not contain a direct summand $\mathbb{Z}$, we can always find another group $G$ (in fact a right-angled Artin group) such that the product formula will not hold.

Remark 5.3. In order to drop the condition that $\chi\left(v_{i}\right) \neq 0$ for all generators $v_{i}$ of $G_{L}$, one has to replace the reduced homology of $L$ by the reduced homology of certain ascending links described in [6]. We will avoid stating a more general version of Theorem 5.2, but note that such a theorem has to consider not only the homology of $L$, but also the homology of certain subcomplexes of $L$. We refer the reader to [6] for details on the subcomplexes involved. 


\section{REFERENCES}

[1] M. Bestvina, N. Brady, Morse theory and finiteness properties of groups, Invent. Math. 129 (1997), 445-470.

[2] R. Bieri, Finiteness length and connectivity length for groups, in: Geometric group theory down under (Canberra, 1996), 9-22, de Gruyter, Berlin, 1999.

[3] R. Bieri, Deficiency and the geometric invariants of a group. With an appendix by Pascal Schweitzer, J. Pure Appl. Algebra 208 (2007), 951-959.

[4] R. Bieri, W. Neumann, R. Strebel, A geometric invariant of discrete groups, Invent. Math. 90 (1987), 451-477.

[5] R. Bieri, B. Renz, Valuations on free resolutions and higher geometric invariants of groups, Comment. Math. Helv. 63 (1988), 464-497.

[6] K.-U. Bux, C. Gonzalez, The Bestvina-Brady construction revisited: geometric computation of $\Sigma$-invariants for right-angled Artin groups, J. London Math. Soc. (2) 60 (1999), 793-801.

[7] R. Gehrke, The higher geometric invariants for groups with sufficient commutativity, Comm. Algebra 26 (1998), 1097-1115.

[8] J. Meier, H. Meinert, L. van Wyk, Higher generation subgroup sets and the $\Sigma$-invariants of graph groups, Comment. Math. Helv. 73 (1998), 22-44.

[9] H. Meinert, The geometric invariants of direct products of virtually free groups, Comment. Math. Helv. 69 (1994), 39-48.

[10] J.-Cl. Sikorav, Points fixes de difféomorphismes symplectiques, intersectiones de sous-variétés lagrangiennes, et singularités de un-formes fermées, Thése, Université Paris-Sud, Centre d'Orsay, 1987.

Department of Mathematical Sciences, University of Durham, Science Laboritories, South Rd, Durham DH1 3LE, United Kingdom

E-mail address: dirk.schuetz@durham.ac.uk 